\title{
Intrinsic neuronal excitability: a role in homeostasis and disease
}

\author{
Aaron J. Camp* \\ Discipline of Biomedical Science, School of Medical Sciences, Sydney Medical School, University of Sydney, Lidcombe, NSW, Australia \\ ${ }^{*}$ Correspondence: aaron.camp@sydney.edu.au
}

\section{A commentary on}

Reconsidering the role of neuronal intrinsic properties and neuromodulation in vestibular homeostasis

by Beraneck, M., and Idoux, E. (2012). Front. Neur. 3:25. doi:10.3389/fneur.2012.00025

The neuronal components of brain circuitry are generally considered "stable" throughout an animals' life; with the exception of times of growth and degeneration that occur during development, aging, or pathology. To maintain this stability, neurons must achieve a balance between changing output to meet new requirements, and keeping output within a satisfactory operating range. This balancing act is done through the combination of synaptic plasticity and changes in intrinsic neuronal excitability. While synaptic plasticity has been the focus of most research into neuronal excitability, growing evidence including that described in the recent review by Beraneck and Idoux (2012), highlights the fact that changes in intrinsic membrane properties also shape the output of single neurons. In their article Beraneck and Idoux (2012) provide evidence for changes in intrinsic excitability of second order vestibular neurons that occur during vestibular compensation - a remarkable example of homeostatic plasticity whereby spontaneous discharge of neurons in the ipsi and contralesional vestibular nuclei is recalibrated following unilateral vestibular damage or labyrinthectomy. While the authors describe clearly how the output of second order vestibular neurons changes with respect to action potential shape and discharge dynamics within the context of vestibular compensation, it is worth noting that the underlying mechanisms of these changes are not isolated to the vestibular system, but appear to be a common feature of homeostatic plasticity in other central nervous system (CNS) structures and during disease. In the following commentary some of the findings highlighted by Beraneck and Idoux are discussed with respect to work on neuronal excitability in other CNS regions.

Beraneck and Idoux (2012) detail evidence to support the hypothesis that changes in background discharge during vestibular compensation of vestibular nucleus neurons appears to be predominately expressed as changes in the excitability of the type B medial vestibular nucleus (MVN) neuron subpopulation. Broadly speaking the changes described in their review can be characterized as those that affect discharge rate (e.g., spike threshold, action potential shape), or those that affect discharge pattern. Importantly, similar modifications of intrinsic membrane properties have also been shown in various CNS pathologies, highlighting their importance as potential therapeutic targets during disease. In the commonly used Pilocarpine model of epilepsy where injection of the muscarinic agonist causes seizures similar to those observed in human temporal lobe epilepsy (Sanabria et al., 2001), downregulation of the slow, non-inactivation voltage-dependent potassium current $\left(I_{\mathrm{h}}\right)$ has been observed in pyramidal neurons of the hippocampus (Jung et al., 2007), as well as entorhinal cortex (Shah et al., 2004). In these structures attenuation of $I_{\mathrm{h}}$ results in hyperpolarization of the resting membrane potential, increased cell resistivity (making the cell more sensitive to small voltage fluctuations), and increased EPSP summation (Shah et al., 2004; Jung et al., 2007).

Similarly, changes in the number of voltage-gated sodium $\left(\mathrm{Na}^{+}\right)$channels have been demonstrated in animal models of chronic pain. In this example, the threshold required to elicit an action potential from dorsal root ganglion (DRG) neurons was reduced following up-regulated expression of voltage- gated $\mathrm{Na}^{+}$channels (Hong et al., 2004; Kirita et al., 2007; Wang et al., 2007). In addition, down-regulation of somatic $I_{\mathrm{A}}$ has been shown in DRG neurons following crush injury of the nerve root (Hu and Gereau, 2003; Hu et al., 2006; Karim et al., 2006; Tan et al., 2006). These changes in DRG neuron intrinsic excitability, presumably underlie the hyperalgesia and allodynia commonly observed in chronic pain models.

While the changes in intrinsic excitability described above can be broadly characterized as those that affect the properties of all-or-none action potentials, experience-dependent changes can also impact on the pattern of neuronal discharge, that is, the number or regularity of action potentials. Beraneck and Idoux (2012) discuss a homeostatic shift toward the phasic type B-like discharge phenotype (including the subset of neurons with lowthreshold "burst" spikes) following chronic attenuation of inhibitory drive, and on the contralesional side following vestibular compensation. Importantly, this change in discharge phenotype is also observed in a number of pathophysiological states (Beck and Yaari, 2008). For example, changes in firing mode toward a phasic phenotype have been reported in models of chronic epilepsy (Sanabria et al., 2001), and in other epilepsy-associated disorders (Baraban and Schwartzkroin, 1995). Further, increases in the number of neurons displaying the burstfiring phenotype have been implicated in other CNS disorders including chronic stress (Okuhara and Beck, 1998), pain (for review see Cummins et al., 2007; Hains and Waxman, 2007), and neuroinflammation (Saab et al., 2004).

What underlies experience-dependent changes in firing mode? In burst-firing neurons such as the phasic type B plus lowthreshold spikes MVN neuron population, a subthreshold depolarization (termed an afterdepolarization; ADP) can exceed action 
potential threshold and elicit a burst of high frequency APs. Changes in the ionic conductances underlying the ADP presumably contribute to the changes in firing mode observed under both homeostatic and pathological conditions. Indeed, blockade of dendritic $I_{A}$ (generated by the fast transient A-type $\mathrm{K}$ channels, and as pointed out by Beraneck and Idoux, 2012, a key signature of the long-term shift toward the type A AP profile in ipsilesional MVN neurons following compensation) increases the amplitude of the ADP in CA1 neuron dendrites beyond that required for action potential generation, and results in a burstfiring phenotype (Magee and Carruth, 1999; Metz et al., 2007). Similarly, there is substantial evidence for the involvement of other voltage-gated channels in regulating the magnitude of the ADP (and firing mode) including $I_{\mathrm{Ca}, \mathrm{T}}$ channels, which, when up-regulated as in the pilocarpine model of epilepsy or in response to ischemia, also cause an increase in ADP magnitude and burst-firing (Yue et al., 2005; Yaari et al., 2007).

Taken together, it is clear that while modifications in intrinsic neuronal excitability outlined by Beraneck and Idoux (2012) are crucial during short and long-term homeostatic phenomenon like vestibular compensation, similar modifications also underlie the excitability of neurons in other CNS structures during pathology. It is worth noting that work on the role of intrinsic neuronal excitability during health and disease is still young when compared with work focused on synaptic mechanisms, and as such represents an exciting avenue for unraveling the mechanisms that contribute to disorders of the CNS, and potentially, novel targets for their treatment.

\section{REFERENCES}

Baraban, S. C., and Schwartzkroin, P. A. (1995). Electrophysiology of CA1 pyramidal neurons in an animal model of neuronal migration disorders: prenatal methylazoxymethanol treatment. Epilepsy Res. 22, 145-156.

Beck, H., and Yaari, Y. (2008). Plasticity of intrinsic neuronal properties in CNS disorders. Nat. Rev. Neurosci. 9, 357-369.

Beraneck, M., and Idoux, Y. (2012). Plasticity of intrinsic neuronal properties in CNS disorders. Nat. Rev. Neurosci. 9, 357-369.

Cummins, T. R., Sheets, P. L., and Waxman, S. G. (2007). The roles of sodium channels in nociception: implications for mechanisms of pain. Pain 131, 243-257.

Hains, B. C., and Waxman, S. G. (2007). Sodium channel expression and the molecular pathophysiology of pain after SCI. Prog. Brain Res. 161, 195-203.

Hong, S., Morrow, T. J., Paulson, P. E., Isom, L. L., and Wiley, J. W. (2004). Early painful diabetic neuropathy is associated with differential changes in tetrodotoxinsensitive and -resistant sodium channels in dorsal root ganglion neurons in the rat. J. Biol. Chem. 279 29341-29350.

Hu, H. J., Carrasquillo, Y., Karim, F., Jung, W. E., Nerbonne, J. M., Schwarz, T. L., and Gereau, R. W. T. (2006). The kv4.2 potassium channel subunit is required for pain plasticity. Neuron 50, 89-100.

Hu, H. J., and Gereau, R. W. T. (2003). ERK integrates PKA and PKC signaling in superficial dorsal horn neurons. II. Modulation of neuronal excitability. J. Neurophysiol. 90, 1680-1688.

Jung, S., Jones, T. D., Lugo, J. N. Jr., Sheerin, A. H., Miller, J.W., D'Ambrosio, R., Anderson, A. E., and Poolos, N. P. (2007). Progressive dendritic HCN channelopathy during epileptogenesis in the rat pilocarpine model of epilepsy. J. Neurosci. 27, 13012-13021.

Karim, F., Hu, H. J., Adwanikar, H., Kaplan, D., and Gereau, R.W.T. (2006). Impaired inflammatory pain and thermal hyperalgesia in mice expressing neuronspecific dominant negative mitogen activated protein kinase kinase (MEK). Mol. Pain 2, 2.

Kirita, T., Takebayashi, T., Mizuno, S., Takeuchi, H., Kobayashi, T., Fukao, M., Yamashita, T., and Tohse, N. (2007). Electrophysiologic changes in dorsal root ganglion neurons and behavioral changes in a lumbar radiculopathy model. Spine 32, E65-E72.

Magee, J. C., and Carruth, M. (1999). Dendritic voltagegated ion channels regulate the action potential firing mode of hippocampal CA1 pyramidal neurons. J. Neurophysiol. 82, 1895-1901.
Metz, A. E., Spruston, N., and Martina, M. (2007). Dendritic D-type potassium currents inhibit the spike afterdepolarization in rat hippocampal CA1 pyramidal neurons. J. Physiol. (Lond.) 581, 175-187.

Okuhara, D. Y., and Beck, S. G. (1998). Corticosteroids influence the action potential firing pattern of hippocampal subfield CA3 pyramidal cells. Neuroendocrinology 67, 58-66.

Saab, C. Y., Craner, M. J., Kataoka, Y., and Waxman, S. G. (2004). Abnormal Purkinje cell activity in vivo in experimental allergic encephalomyelitis. Exp. Brain Res. 158, 1-8.

Sanabria, E. R., Su, H., and Yaari, Y. (2001). Initiation of network bursts by $\mathrm{Ca}^{2+}$-dependent intrinsic bursting in the rat pilocarpine model of temporal lobe epilepsy. J. Physiol. (Lond.) 532, 205-216.

Shah, M. M., Anderson, A. E., Leung, V., Lin, X., and Johnston, D. (2004). Seizure-induced plasticity of $h$ channels in entorhinal cortical layer III pyramidal neurons. Neuron 44, 495-508.

Tan, Z. Y., Donnelly, D. F., and LaMotte, R. H. (2006). Effects of a chronic compression of the dorsal root ganglion on voltage-gated $\mathrm{Na}^{+}$and $\mathrm{K}^{+}$currents in cutaneous afferent neurons. J. Neurophysiol. 95, 1115-1123.

Wang, J. G., Strong, J. A., Xie, W., and Zhang, J. M. (2007). Local inflammation in rat dorsal root ganglion alters excitability and ion currents in small-diameter sensory neurons. Anesthesiology 107, 322-332.

Yaari, Y., Yue, C., and Su, H. (2007). Recruitment of apical dendritic T-type $\mathrm{Ca}^{2+}$ channels by backpropagating spikes underlies de novo intrinsic bursting in hippocampal epileptogenesis. J. Physiol. (Lond.) 580, 435-450.

Yue, C., Remy, S., Su, H., Beck, H., and Yaari, Y. (2005). Proximal persistent $\mathrm{Na}^{+}$channels drive spike afterdepolarizations and associated bursting in adult CA1 pyramidal cells. J. Neurosci. 25, 9704-9720.

Received: 05 March 2012; accepted: 19 March 2012; published online: 03 April 2012.

Citation: Camp AJ (2012) Intrinsic neuronal excitability: a role in homeostasis and disease. Front. Neur. 3:50. doi: 10.3389/fneur.2012.00050

This article was submitted to Frontiers in Neuro-otology, a specialty of Frontiers in Neurology.

Copyright (C) 2012 Camp. This is an open-access article distributed under the terms of the Creative Commons Attribution Non Commercial License, which permits non-commercial use, distribution, and reproduction in other forums, provided the original authors and source are credited. 